\title{
No REST for healthy beta cells
}

\section{G. Thiel • F. Schuit}

Published online: 21 May 2008

(C) Springer-Verlag 2008

Keywords Beta cell · Differentiation · Gene silencing . Exocytosis · Insulin release $\cdot$ Neuronal traits .

RE-1 silencing transcription factor $\cdot R E S T$ expression .

Transcriptional repressor

$\begin{array}{ll}\text { Abbreviations } \\ \text { CX36 } & \text { connexin } 36 \\ \text { HP1 } & \text { heterochromatin protein-1 } \\ \text { RE-1 } & \text { repressor element 1 } \\ \text { REST } & \text { RE-1 silencing transcription factor } \\ \text { SNAP25 } & \text { synaptosomal-associated protein } 25 \\ \text { SYT } & \text { synaptotagmin }\end{array}$

In this issue of Diabetologia, the paper by Martin et al. [1] has provided new insights into an interesting area of beta cell research. The field started about a decade ago [2], with the discovery of a molecular explanation for the fact that beta cells and neurons share many phenotypic traits, such as electrical excitability, production and secretion of amines (e.g. $\gamma$-aminobutyric acid), production of neurotrophin receptors and neuron-specific transcription factors. Part of the molecular explanation for this phenotypic similarity is based upon negative regulation, and a key player in this process is the

\footnotetext{
G. Thiel

Department of Medical Biochemistry and Molecular Biology, University of Saarland,

Hamburg, Germany

F. Schuit $(\square)$

Department of Molecular Cell Biology, Gene Expression Unit, Katholieke Universiteit Leuven,

Herestraat 49, Mailbox 901, 3000 Leuven, Belgium

e-mail: frans.schuit@med.kuleuven.be
}

transcriptional repressor known as transcriptional repressor element 1 (RE-1) silencing transcription factor (REST). This repressor is known to prevent the transcription of neuronal genes in most cell types [3] other than beta cells and neurons, which are almost completely devoid of the REST protein $[2,3]$. In this commentary we will provide a brief review of the biological effects of REST, followed by a discussion of the work of Martin et al. [1].

A large number of studies indicate that REST plays a key role in the establishment of the neuronal phenotype [4] and functions as a transcriptional repressor of neuronal genes in non-neuronal tissues. Genes targetted by REST encode neuronal receptors, ion channels, neuropeptides, synaptic vesicle proteins, transcription factors and adhesion molecules, underlining the important role of REST in controlling the neuronal phenotype. The widespread production of REST protein in non-neuronal tissues is in good agreement with the role of REST as a negative regulator of neuronspecific gene transcription. Thus, a negative regulatory mechanism, involving strong expression of REST in nonneuronal cells and marginal expression of REST in neurons, controls the establishment of the neuronal phenotype [3]. Recently, REST has been shown to regulate both the transition from pluripotent to neural stem/progenitor cells and from progenitor cells to mature neurons [5]. In addition, REST controls the differentiation of human neural stem cells along the neuronal lineage [6]. However, the current view of REST function in the nervous system is mainly based on experiments performed with cultured cells, as currently there is no mouse model available that constitutively expresses REST in the nervous system.

On a molecular level, REST recruits histone deacetylases and methyltransferases to its target genes, indicating that modulation of the chromatin structure is crucial for neuronal 
gene transcription $[7,8]$. The recruitment of histone deacetylases by REST results in removal of acetyl groups from the core histones. Consequently, the neuronal genes are embedded into more tightly packed chromatin that is inaccessible to the transcriptional machinery. The silencing activity of REST has also been shown to involve histone methylation of target genes. REST recruits the histone dimethylase G9a to neuronal genes, which triggers the methylation of lysine residue 9 of histone H3 [9]. Methylation of $\mathrm{H} 3 \mathrm{~K} 9$ provides a high-affinity binding site for heterochromatin protein-1 (HP1). Oligomerisation of HP1 following binding causes the formation of a higher ordered chromatin compaction, known as heterochromatin, which is in a transcriptionally silent state.

The cellular concentration of REST is important for neuronal gene transcription. Accordingly, downregulation of REST production during neurogenesis is necessary for proper neuronal development. The concentration of REST protein is extremely low in the nuclei of neurons. Neuronal gene chromatin has an open configuration in these cells, allowing transcriptional activators to bind and initiate transcription. Thus, transcription of neuronal genes is the result of a relief of repression. Furthermore, REST is not produced in the normal endocrine pancreas [2, 10]. Accordingly, pancreatic beta cells express neuronal genes encoding synaptic vesicle proteins, neurotransmitters and neurotransmitter-synthesising enzymes, suggesting that downregulation of REST production in beta cells triggers a de-repression of REST-controlled genes in these cells. However, the REST concentration is not the only critical parameter for REST-regulated gene transcription. Chromatin remodelling, including differentiation-dependent changes in the histone methylation pattern, occurs during development and is required for cell type-specific gene transcription. Thus, both the cellular concentration of REST and the cell type-specific structure of the chromatin determines whether REST-target genes are transcribed. In this context, it is interesting that the REST target genes encoding synaptophysin, a major synaptic vesicle protein, and connexin 36 (CX36) have an open chromatin structure in $\beta \mathrm{TC} 3$ insulinoma cells [10].

To further study the function of the physiologically very low expression of REST in pancreatic beta cells, Martin et al. [1] used genetically modified mice (referred to as RIP-REST throughout the remainder of this commentary) in which expression of human REST mRNA was selectively induced in beta cells under control of the rat Ins 2 promoter. As expected, REST protein accumulated in the nuclei of the transgenic animals. Interestingly, such mice develop impaired glucose tolerance as young adults, and this intolerace was explained by significantly reduced plasma insulin levels. In their search for an explanation for this phenotype, the authors initially measured the insulin content of the RIP-
REST adult pancreases, reporting a 50\% reduction relative to that in wild-type animals. Futhermore, beta cell number per pancreas was around 30\% lower. Though interesting in itself, this type of reduction seemed insufficient to explain the glucose intolerance. Furthermore, the ultrastructure of beta cells appeared normal, with healthy numbers of typical dense core granules present. So another defect in the beta cell was suspected. The next experiment was to isolate pancreatic islets and study insulin release in perifusion experiments. As is elegantly shown in the paper, the basal rate of insulin release from the RIP-REST islets is normal, but both the first and second phase of insulin release induced by $20 \mathrm{mmol} / \mathrm{l}$ glucose are significantly decreased compared with those in wild-type islets, even when corrected for the lower islet insulin content. Interestingly, this secretory defect was mimicked in an in vitro model of acute REST expression, which involved adenoviral transfer of the gene into non-transgenic wild-type islets, suggesting that REST mainly alters the phenotype of differentiated beta cells, rather than influencing the decisions on the way towards beta cell differentiation.

This idea was further examined in the glucose-responsive cell line INS-1E transfected with the REST-encoding adenovirus (Ad-REST). Compared with control cells, both the triggering and amplifying pathways of glucose-stimulated insulin secretion were attenuated. Depolarising concentrations of $\mathrm{KCl}$ were also less effective as a secretagogue in Ad-RESTtreated cells, and both insulin content and stimulation-induced calcium levels were the same in control and Ad-REST cells. Together, the data suggested that REST has a negative influence on the exocytotic machinery. The logical next step was to examine the expression of genes that are relevant to the exocytotic process. As in other secreting cells, many different proteins on the secretory vesicle membrane and plasma membrane are involved in signal-induced granule fusion and release of insulin into the extracellular space (reviewed in [11]). Martin et al. [1] used bioinformatic tools, screening all predicted REST-reponsive genes for those that act at the level of exocytosis and contain a promoter element that resembles the known 21 bp human RE-1. This revealed a handful of target genes likely to account for the transgenic phenotype, notably the $N$-ethylmaleimide-sensitive factor attachment protein receptor (SNARE) protein known as synaptosomal-associated protein 25 (SNAP25) and the $\mathrm{Ca}^{2+}$ sensitive synaptotagmins IV and VII (SYT4 and SYT7). Using DNA isolated from INS-1E cells, binding of REST to the promoter of these genes was shown via chromatin immunoprecipitation with anti-REST antiserum. In line with this result, INS-1E cells expressing REST were repressed in terms of the transcription of the same exocytosis-related genes, as illustrated by northern blots and real-time PCR experiments. For SNAP25 and SYT4 further evidence for REST repression was found at the protein level. The main 
conclusion drawn by the authors of the paper [1] is that, in adult differentiated beta cells, REST expression should be avoided, because the repressor interferes with full expression of genes encoding important proteins of the exocytotic machinery.

The study by Martin et al. [1] is also stimulating further research, as it leaves us with a number of new questions. The decreases in pancreatic insulin content and beta cell number in the transgenic model remain unexplained. REST is crucial for the differentiation of neuronal [5] and heart progenitor cells [12]. Moreover, it has been suggested that Pax4 expression in the developing pancreas may be regulated by REST [13]. Thus, it seems possible that, in RIP-REST mice, forced REST expression slows down beta cell maturation or postnatal beta cell replication. Along this line, transgenic models other than RIP-REST could be of interest, for instance by using promoter sequences that induces REST expression at an earlier endocrine or pancreatic progenitor stage.

It is also important to dissociate the influence of REST on Cx36 expression from its effects on the exocytotic machinery. In another paper, the same authors have reported that the beta cell-selective gap junction protein Cx36 [14] is important for normal glucose-induced insulin release [15]. As the $C x 36$ gene is regulated by REST in beta cells $[10,16]$, in the present transgenic model [1] it is not clear to what extent the downregulation of $C x 36$ expression accounts for the reduced insulin release and glucose intolerance.

In addition to the genes encoding SNAP25, SYT4 and SYT7, the genes for chromogranin B and secretogranin II are also regulated by REST in insulinoma cells [10]. The granins are abundant in endocrine and neuroendocrine cells, and are involved in the formation of secretory vesicles and in the aggregation-mediated sorting of peptide hormones into secretory granules (reviewed in [17]). Along this line, a recent study with PC12 pheochromocytoma cells showed that REST-regulated gene transcription is crucial for the maintenance of the neurosecretory process and the properties of large dense core vesicles [18]. When all these data are considered together with those of Martin et al. [1], a picture emerges of how the downregulation of REST production is essential for many proteins involved in the exocytotic process.

It would be interesting to compare the epigenetic signature of REST-regulated genes in neurons and beta cells. This would include an analysis of the molecular mechanism responsible for the generation of an open chromatin configuration of REST-responsive genes, as has been shown for $C x 36$ and the synaptophysin genes [10].

A final important question is: what physiological mechanism keeps REST expression low in maturing beta cells? Is it the same mechanism as used by neurons? A repressor used both by neurons and beta cells to prevent
REST transcription could be the explanation. Furthermore, it would be interesting to analyse the impact of microRNAs, in particular, miR124, on the REST protein content of beta cells [19]. In addition to REST, expression of the genes encoding low- $K_{\mathrm{m}}$ hexokinases [20], lactate dehydrogenase [21], and monocarboxylate transporter 1 [22] is also disallowed in healthy beta cells. An emerging concept is that avoidance of transcription of disallowed genes may be as important for the specific beta cell phenotype as expression of beta cell-specific genes [23]. Violation of this disallowance of the gene encoding monocarboxylate transporter 1 (also known as SLC16A1) in humans causes exercise-induced hyperinsulinism and hypoglycaemia [24]. The work of Martin et al. [1] predicts that beta cell-selective violation of REST disallowance may predispose to human diabetes.

Duality of interest The authors declare that there is no duality of interest associated with this manuscript.

\section{References}

1. Martin D, Allagnat F, Chaffard G et al (2008) Functional significance of REST target genes in pancreatic beta cells. Diabetologia DOI 10.1007/s00125-008-0984-1

2. Atouf F, Czernichow P, Scharfmann R (1997) Expression of neuronal traits in pancreatic beta cells. Implication of neuron-restrictive silencing factor/repressor element silencing transcription factor, a neuron-restrictive silencer. J Biol Chem 272:1929-1934

3. Chong JA, Tapia-Ramirez J, Kim S et al (1995) REST: a mammalian silencer protein that restricts sodium channel gene expression to neurons. Cell 80:949-957

4. Thiel G, Hohl M (2006) RE-1 silencing transcription factor (REST) - regulation of neuronal gene expression via modification of the chromatin structure. In: Thiel G (ed) Transcription factors in the nervous system - development, brain function and disease. Wiley, Germany, pp 113-128

5. Ballas N, Grunseich C, Lu DD, Speh JC, Mandel G (2005) REST and its corepressors mediate plasticity of neuronal gene chromatin throughout neurogenesis. Cell 121:645-657

6. Ekici M, Hohl M, Schuit F, Martínez-Serrano A, Thiel G (2008) Transcription of genes encoding synaptic vesicle proteins in human neural stem cells: chromatin accessibility, histone methylation pattern, and the essential role of REST. J Biol Chem 283:9257-9268

7. Thiel G, Lietz M, Hohl M (2004) How mammalian transcriptional repressors work. Eur J Biochem 271:2855-2862

8. Ooi L, Wood IC (2007) Chromatin crosstalk in development and disease: lession from REST. Nat Rev Genet 8:544-554

9. Roopra A, Qazi R, Schoenike B, Daley TJ, Morrison JF (2004) Localized domains of G9a-mediated histone methylation are required for silencing of neuronal genes. Mol Cell 14:727-738

10. Hohl M, Thiel G (2005) Cell type-specific regulation of RE-1 silencing transcription factor (REST) target genes. Eur J Neurosci 22:2216-2230

11. Jahn R, Lang T, Südhof TC (2003) Membrane fusion. Cell 112:519-533

12. Kuwahara K, Saito Y, Takano M et al (2003) NRSF regulates the fetal cardiac gene program and maintains normal cardiac structure and function. EMBO J 22:6310-6321 
13. Kemp DM, Lin JC, Habener JF (2003) Regulation of Pax4 paired homeodomain gene by neuron-restrictive silencer factor. J Biol Chem 278:35057-35062

14. Serre-Beinier V, Le Gurun S, Belluardo N et al (2000) Cx36 preferentially connects beta-cells within pancreatic islets. Diabetes 49:727-734

15. Le Gurun S, Martin D, Formenton A et al (2003) Connexin-36 contributes to control function of insulin-producing cells. J Biol Chem 278:37690-37697

16. Martin D, Tawadros T, Meylan L et al (2003) Critical role of the transcriptional repressor neuron-restrictive silencer factor in the specific control of connexin36 in insulin-producing cell lines. J Biol Chem 278:53082-53089

17. Taupenot L, Harper KL, O’Connor DT (2003) The chromograninsecretogranin family. N Engl J Med 348:1134-1149

18. D'Alessandro R, Klajn A, Stucchi L, Podini P, Malosio ML, Meldolesi J (2008) Expression of the neurosecretory process in pc12 cells is governed by rest. J Neurochem 105:1369-1383
19. Makeyev EV, Maniatis T (2008) Multilevel regulation of gene expression by microRNAs. Science 319:1789-1790

20. Schuit F, Moens K, Heimberg H, Pipeleers D (1999) Cellular origin of hexokinase in pancreatic islets. J Biol Chem 274:32803-32809

21. Sekine N, Cirulli V, Regazzi R et al (1994) Low lactate-dehydrogenase and high mitochondrial glycerol phosphate dehydrogenase in pancreatic beta-cells - potential role in nutrient sensing. J Biol Chem 269:4895-4902

22. Zhao C, Wilson MC, Schuit F, Halestrap AP, Rutter GA (2001) Expression and distribution of lactate/monocarboxylate transporter isoforms in pancreatic islets and the exocrine pancreas. Diabetes 50:361-366

23. Quintens R, Hendrickx N, Lemaire K, Schuit F (2008) Why expression of some genes is disallowed in beta cells. Biochem Res Transact 36:300-305

24. Otonkoski T, Jiao H, Kaminen-Ahola N et al (2007) Physical exerciseinduced hypoglycemia caused by failed silencing of monocarboxylate transporter 1 in pancreatic beta cells. Am J Hum Genet 81:467-474 